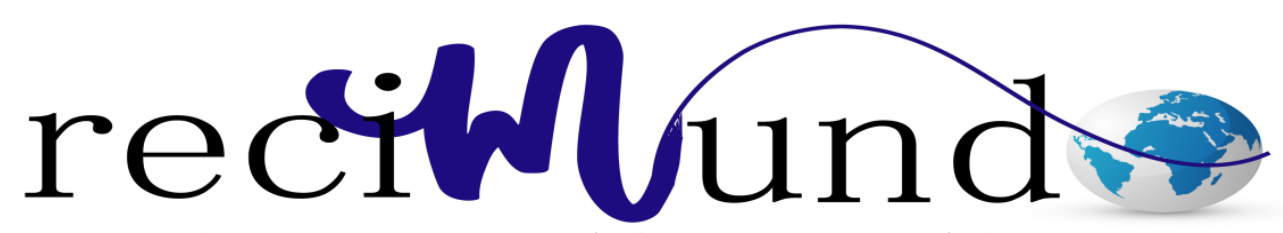

Revista Científica Mundo de la Investígación y el Conocimiento

\author{
Milton Marcos González Santos a ; José Bladimir Guarnizo Delgado b; Wagner \\ Enrique Ortega Arcos c; Vilma Maribel García González d
}

Arte y Educación

Revista Científica Mundo de la Investigación y el Conocimiento. Vol. 1 núm., 5, diciembre, 2017, pp. 101-1022

DOI: $10.26820 /$ recimundo/1.5.2017.1011-1022

Editorial Saberes del Conocimiento

a. Magister en Gerencia Educativa; Diploma Superior en Gestión y Planificación Educativa; Magister en Periodismo; Licenciado en Ciencias de la Comunicación Social; Docente, Carrera de Comunicación. Universidad Estatal Península de Santa Elena. UPSE; migonsa15@gmail.com

b. Magister en Comunicación Corporativa; Especialista en Imagen Corporativa; Maestría en Gerencia Educativa; Diploma Superior en Gestión y Planificación Educativa; Diploma Superior en Gerencia con Programación Neurolingüística; Diploma Superior en Evaluación de la Calidad de las Instituciones de Educación Superior; Diploma Superior en Diseño de Proyectos; Licenciado en Comunicación Social con mención en Televisión; Carrera de Comunicación Social Universidad Estatal de Bolívar UEB; jblady guarnizo@ yahoo.es

c. Magister en Comunicación Corporativa; Especialista en Imagen Corporativa; Licenciado en Comunicación Social; Universidad Técnica de Ambato UTA; wagnerortega10@gmail.com

d. Licenciada en Comunicación Social; Universidad Internacional de la Rioja UNIR; mabelita.garcía11800@gmail.com 


\section{Arte y Educación}

Vol. 1, núm. 5., (2017)

Milton Marcos González Santos; José Bladimir Guarnizo Delgado; Wagner Enrique Ortega Arcos; Vilma Maribel García González

\section{RESUMEN}

Se plantea en el presente artículo revisar la relación que debe existir entre el arte y la educación desde la mirada de varios autores. Se considera a las artes como el modelo de integración entre el saber técnico, científico y creativo. Mediante la revisión bibliográfica de expertos en el tema del Arte y la educación, considerando las influencias del pensamiento crítico, se invita a reflexionar acerca de nuevas perspectivas acerca del hecho educativo, rompiendo con la racionalidad instrumental predominante en el modelo educativo hegemónico. Los autores revisados consideran al arte como una herramienta fundamental en la educación como instrumento para transformar y construir una conciencia crítica, reflexiva y una sensibilidad del ser. Se revisan las aportaciones concretas de las narrativas de autores tales como Saramago y Camus como ejemplo de como el arte estimula la liberación de la imaginación a través de imágenes y metáforas, donde se necesite de la implicación de la imaginativa de las artes. Así mismo se plantea que, a través de un cuadro, una danza o una música se logran ampliar las sensibilidades de las formas, se podrá despertar la imaginación que es la función real del arte y esto haría despertar el sentido. Por otra parte, el arte contribuiría a preparar a los sujetos para una convivencia humana solidaria, cooperativa. Se concluye en que definitivamente debe incorporarse el arte en la educación como medio para formar sujetos críticos, integrales, transformar la escuela y desarrollar la cultura como esa capacidad de desarrollar la imaginación para hacer las cosas mejor, con la aspiración de vivir en un mundo mejor.

Palabras claves: Arte, educación, pensamiento crítico, imaginación. 
Arte y Educación

Vol. 1, núm. 5., (2017)

Milton Marcos González Santos; José Bladimir Guarnizo Delgado; Wagner Enrique Ortega Arcos; Vilma Maribel García González

\begin{abstract}
It is proposed in this article to review the relationship that should exist between art and education from the perspective of several authors. The arts are considered as the integration model between technical, scientific and creative knowledge. Through the bibliographic review of experts in the subject of art and education, considering the influences of critical thinking, we invite reflection on new perspectives about the educational fact, breaking with the predominant instrumental rationality in the hegemonic educational model. The authors reviewed consider art as a fundamental tool in education as an instrument to transform and build a critical, reflective awareness and sensitivity of being. The specific contributions of the narratives of authors such as Saramago and Camus are reviewed as an example of how art stimulates the liberation of the imagination through images and metaphors, where the involvement of the imaginative of the arts is needed. It is also suggested that, through a painting, a dance or a music, the sensibilities of the forms can be enlarged, the imagination that is the real function of art can be reawakened and this would awaken the meaning. On the other hand, art would help to prepare subjects for a solidary, cooperative human coexistence. It concludes that art should definitely be incorporated into education as a means to form critical, integral subjects, transform the school and develop culture as that capacity to develop the imagination to do things better, with the aspiration to live in a better world .
\end{abstract}

Key words: Art, education, critical thinking, imagination. 


\section{Arte y Educación}

Vol. 1, núm. 5., (2017)

Milton Marcos González Santos; José Bladimir Guarnizo Delgado; Wagner Enrique Ortega Arcos; Vilma Maribel García González

\section{Introducción.}

El presente el artículo plantea abordar la relación que debe existir entre arte y educación para transformar y educar al ser. Interesa específicamente el rescate de las condiciones humanas accediendo a la consciencia, sensibilidad y reflexión del pensamiento. Se considera de importancia cardinal plantearse el superar el paradigma educativo tradicional, imbuido en la racionalidad instrumental, mediante la incorporación vinculante de la práctica de las artes en sus diferentes disciplinas como medio para estimular y posibilitar la sensibilidad y la imaginación, en aras de construir sujetos activos para la transformación social. Esto implica necesariamente la formación de los facilitadores en las técnicas pertinentes a la incorporación del arte en la educación.

\section{Aspectos conceptuales}

Desde la racionalidad instrumental predominante, el sistema educativo tendría como misión la de satisfacer las necesidades técnicas y económicas de la sociedad, tendiendo a convertir a los educandos en piezas a ser incorporadas en la gran maquinaria de la economía nacional. En este contexto "reestructuración escolar significa, en muchos sentidos, romper con los viejos modelos cuantitativos" (Green, 2005, p.36), y qué mejor instrumento para la transformación educativa que el arte, en tanto posibilitador de liberación de la imaginación, en las prácticas cotidianas de los procesos educativos en tanto interacciones cotidianas entre los facilitadores y los educandos.

En este sentido, se debe considerar que las artes pueden ser un modelo de integración entre el saber científico, técnico y creativo. Debe permitir la integridad del ser humano, 
Arte y Educación

Vol. 1, núm. 5., (2017)

Milton Marcos González Santos; José Bladimir Guarnizo Delgado; Wagner Enrique Ortega Arcos; Vilma Maribel García González

desarrollando una habilidad metacognitiva para reflexionar y la capacidad de percibir quién es, de dónde viene, hacia dónde va, lo que hace, que debe hacer, como autocriticarse para cambiar y transformar para convivir y vivir en equilibrio con él mismo, con los demás y el entorno.

Esto implica reflexionar hacia una nueva perspectiva de la educación para que no siga dedicándose solo a reproducir y reforzar los valores que la sociedad demanda, porque su intencionalidad debería ser formar un sujeto integral capaz de autorrealizarse, es decir, que se encuentre a sí mismo y tenga amor por los demás (Beuchot, 2004). El ser humano, por su naturaleza social, la condición de su sobrevivencia depende de su vínculo afectivo con los demás y la construye a través de una simbolización.

Este símbolo es lo que le permite formar un lazo con los demás, tener una cohesión, encontrar sentido a las cosas, comprender las realidades, recrearlas y dar su versión de la misma. Por lo que la educación tiene como papel importante el profundizar en la realización del sujeto, así como romper con los paradigmas de una educación solo instrumentalista, que se reduce solo a la enseñanza de conocimiento, y al seguir instrucciones.

En ese mismo orden de ideas, algunos teóricos críticos como Adorno, Horkheimer, Marcuse, Maturana, Giroux, Grascmi plantean que se debe transformar y construir una conciencia crítica, reflexiva y una sensibilidad del ser. Por lo tanto, se debe transformar las estructuras educativas, impulsada dicha transformación por múltiples disciplinas, entre las que, de manera principal, debe estar el arte como una práctica cultural y política para la conciencia social, que fomente la capacidad de los sujetos a situarse a sí mismos dentro de sus conexiones 


\section{Arte y Educación}

Vol. 1, núm. 5., (2017)

Milton Marcos González Santos; José Bladimir Guarnizo Delgado; Wagner Enrique Ortega Arcos; Vilma Maribel García González

históricas y a construir opciones con un futuro, es decir, con una utopía que pueda definirse en términos más humanos, justos y esperanzados.

Los autores antes mencionados realizan una crítica a la razón instrumental y proponen una pedagogía radical que sea capaz de desarrollar una epistemología de la conciencia crítica, que pueda dar herramientas teóricas útiles para cuestionar la realidad, y de esta manera plantearse un mundo diferente, en el que se aprenda una forma de vida llena de paz, amor, esperanza, solidaridad, respeto, cooperación.

En concordancia con esas perspectivas, Greene (2005) argumentó en su teoría las funciones de la enseñanza del arte, en el sentido de propiciar el desarrollo habilidades, la mejora del rendimiento académico, liberando la imaginación para que los sujetos observen las cosas como si pudieran ser de otro modo. Para ello debe enseñarse al estudiante a prestar atención a las formas, las pautas, los sonidos, los ritmos, los contornos y las líneas e incluso ayudarlos a crear obras de arte, porque estas pueden nutrir el crecimiento e inventiva de personas y además pueda solucionar los problemas.

Porque el arte como tal ofrece la luz, el descubrimiento, por lo que se requiere que se tome el tiempo para enseñar y practicar la autorreflexión, acerca de si somos capaces de percibirnos a nosotros mismos como interrogadores, como creadores de sentido, como personas implicadas en la construcción y reconstrucción de realidades junto a las personas que nos rodean.

Una de las teorías que Green (2005) compartió en sus ensayos es demostrar que debemos partir de enseñar a liberar la imaginación apoyándonos de las diferentes disciplinas del arte, con 
Arte y Educación

Vol. 1, núm. 5., (2017)

Milton Marcos González Santos; José Bladimir Guarnizo Delgado; Wagner Enrique Ortega Arcos; Vilma Maribel García González

el propósito de que podamos organizar un mundo coherente, capaces de mirar a través de esos extraños, de oír con sus mismos oídos, e introducirnos en el tejido social.

Esto implica una reestructuración de la educación y el rompimiento con el modelo cuantitativo, porque la educación si es vista desde lo humanista debe preocuparse por la búsqueda de la libertad intelectual y de expresión. Que los jóvenes y las jóvenes sean capaces de plantearse su propias preguntas, expliquen sus propias historias, que logren ser profesionales críticos y autorreflexivos. Es allí cuando se nos revela la importancia que tiene el pensamiento imaginativo, que se aplique a las posibles formas de organización social con la intencionalidad de que las cosas sean de otra manera. Es así, porque a través de las metáforas el hombre puede evocar situaciones que dificultan la construcción de la comunidad y a restablecer la dignidad humana.

Por ejemplo, en la obra de Saramago (2004), el Ensayo sobre la ceguera, la agnosis consiste en la incapacidad de reconocer lo que se ve, o el desconocimiento e interrupción de la capacidad de reconocer estímulos, sin haber deficiencias o alteración de la percepción, lenguaje o intelecto. En su obra describe una ceguera colectiva en la que la especie humana debe aprender a sobrevivir. Así mismo, establece cómo otros hombres eran capaces de aprovecharse de las circunstancias para robar, valiéndose de las necesidades de los pobres, como aflora el egoísmo, y como a veces dejamos de ver los problemas que vivimos cuando no buscamos soluciones a ellos. Saramago nos ofrece en su obra narrativa como el miedo nos paraliza y no hace tener ceguera colectiva. 


\section{Arte y Educación}

Vol. 1, núm. 5., (2017)

Milton Marcos González Santos; José Bladimir Guarnizo Delgado; Wagner Enrique Ortega Arcos; Vilma Maribel García González

Por su parte, en la obra de Camus (2002), la Peste, está presente la metáfora en su narrativa para describir como las enfermedades inmovilizan a las personas, más aun cuando son colectivas, se resignan, aíslan o desesperan, pensando que es incurable. Sin embargo, en el desarrollo, después de la lucha contra la peste, se desarrolla el desenlace cuando el médico reacciona y toma conciencia que no puede aceptar la plaga y se da cuenta que lo que debe hacer es reorganizar a la gente en brigadas sanitarias para combatir la peste, demostrándonos, en su metáfora, que la plaga no es más que las evasiones de los problemas complejos, de ver las dificultades humanas y buscar las soluciones.

Desde la perspectiva de Greene (2005), eso sucede cuando los seres humanos presentan obstáculos en el pensamiento imaginativo y es allí donde se necesita de la implicación de la imaginativa de las artes. Citando a Camus (1948) y a Arendt (1958), este autor plantea que la conciencia y la apertura al mundo son las que permiten darse cuenta de las posibilidades alternativas y mostrarse conforme con las realidades, porque cuando se lee y se deja que se induzca un nuevo conjunto de interrogantes, que haría pensar cómo deberían ser las cosas.

En ese mismo orden de ideas, Greene (2005) reafirma por qué su defensa de las artes y aclara que se debe reconocer que los hechos que componen las experiencias estéticas ocurren dentro (y por medio) de las transacciones del entorno porque sitúan en el tiempo y el espacio.

Al igual que las obras literarias, otras formas artísticas hacen recobrar la espontaneidad perdida. Porque cuando a través de un cuadro, una danza o una música se logra ampliar las sensibilidades de las formas, se podrá despertar la imaginación que es la función real del arte y 
Arte y Educación

Vol. 1, núm. 5., (2017)

Milton Marcos González Santos; José Bladimir Guarnizo Delgado; Wagner Enrique Ortega Arcos; Vilma Maribel García González

esto haría despertar el sentido, percibirse a si mismos como interrogadores, recrearse en la construcción o reconstrucción de realidades.

Finalmente se debe reconocer que las artes nos pueden ayudar a crecer, a tener inventiva y a solucionar problemas. A través de ellas se puede conseguir la libertad humana.

Ambos autores, Camus y Saramago, así como muchos otros, hacen entrar en un mundo vivido que tal vez no se ha vivido, pero que ha estado presente y que debe sensibilizar y hacer reflexionar sobre las historias que reconstruyeron una realidad y despierta la imaginación.

¿Por qué fomentar el arte en la escuela?

La razón por la que se debe fomentar el arte en la educación, es porque es un factor determinante que inicia el proceso del desarrollo evolutivo, sensitivo e intelectual del estudiante y del profesor. Además constituye un medio para comunicarse y expresarse en pensamientos y sentimientos. Si el profesor implementa el arte en el aula motiva a sus estudiantes a despertar la creatividad, la expresión y el desarrollo de la apreciación estética; elementos que logran integrar la personalidad del alumno, en sí mismos. El arte como práctica de la creatividad resultaría de forma más objetiva si este proceso llevase un planeamiento teórico práctico que considera un equilibrio en cuanto a pensamiento/cuerpo, razonamiento/sensibilidad.

Ahora bien, no es solamente justificar la enseñanza del arte, sino entender que el arte genera una expansión de la capacidad sensitiva, y por ende, de nuestro entendimiento, tal como lo establece Silva (2011) el verdadero arte consiste siempre en la creación de formas nuevas, que amplían el horizonte psicológico y cultural de los hombres y los pueblos. Una revolución social 


\section{Arte y Educación}

Vol. 1, núm. 5., (2017)

Milton Marcos González Santos; José Bladimir Guarnizo Delgado; Wagner Enrique Ortega Arcos; Vilma Maribel García González

necesita de este ingrediente fundamental para poder realizar la transformación de las conciencias, necesaria para la aceptación del nuevo orden y nuevo estilo vital

Maturana (2003) establece que para ello se debe propiciar un estado de conciencia, responsabilidad y autoridad para las decisiones, para así lograr elevar las posibilidades de crear, transformar e innovar en todos los medios existentes. Es decir, que desde la participación de la comunidad, se puede lograr que la acción educativa desarrolle estrategias de transformación, con las que se aprenda a ser humano, y que esta sea capaz de poner fin a las desigualdades sociales, con una concepción que active las emociones y que conlleve a que el hombre aprenda a vivir en armonía con la naturaleza, consigo mismo y con los demás.

Para el análisis y comprensión de la importancia de la incorporación del estudio y desarrollo del arte nos apoyamos de la teoría de Maturana (2003) en la que establece que la escuela debe configurarse como un componente de la comunidad, de manera que logre convivir con cada persona y logre entender el cúmulo de influencias culturales, que se trasmiten de generación en generación por las interacciones sociales, donde el lenguaje juega un papel preponderante. Porque sólo a través del él los seres humanos hemos representado nuestro desarrollo y nuestra cultura, esa capacidad de desarrollar la imaginación para hacer las cosas mejor, con la aspiración de vivir en un mundo mejor.

La educación debe ser una actividad placentera, llena de amor y emociones, buscando la aceptación de la convivencia de las comunidades y respetando la diversidad humana. Esto hace pensar que también se debe formar y reeducar a los educadores para que enseñen desde el amor y sean capaces de aflorar sus emociones y sentimientos para enseñar y ser ejemplo entro lo que se 
Arte y Educación

Vol. 1, núm. 5., (2017)

Milton Marcos González Santos; José Bladimir Guarnizo Delgado; Wagner Enrique Ortega Arcos; Vilma Maribel García González

es y lo que se hace, y la aceptación de sí mismo y de los demás. Que sea él un guía, un orientador, un líder por el desarrollo y crecimiento de la afectividad, la solidaridad, de manera de minimizar en las escuelas tanta violencia, agresividad, maltrato, negación en todos los niveles, reproducido de alguna manera también por los docentes cuando interactúan con sus estudiantes.

\section{A manera de conclusión:}

La posibilidad real de que la educación contribuya de manera significativa a incorporar a los sujetos a la solución de los grandes problemas de las comunidades, del país y del mundo actual, pasan por trascender el modelo educativo fundamentado en la racionalidad instrumental, con su énfasis en los aspectos cuantitativos y para quienes los sujetos son piezas de una gran maquinaria económica.

$\mathrm{El}$ arte, en tanto componente esencialmente humano del ser, ofrece las posibilidades y potencialidades para estimular la liberación de la imaginación de los sujetos de la educación, convirtiéndolos en seres reflexivos, críticos, proactivos, con capacidades para elevarse por encima de las apariencias borrosas de la cotidianeidad y desarrollando entonces las potencialidades para proponer y ejecutar soluciones creativas a las problemáticas. Definitivamente debe incorporarse el arte en la educación como medio para formar sujetos críticos, integrales, transformar la escuela y desarrollar la cultura como esa capacidad de desarrollar la imaginación para hacer las cosas mejor, con la aspiración de vivir en un mundo superior. 


\section{Arte y Educación}

Vol. 1, núm. 5., (2017)

Milton Marcos González Santos; José Bladimir Guarnizo Delgado; Wagner Enrique Ortega Arcos; Vilma Maribel García González

\section{Bibliografía.}

Beuchot, M. (2004). Antropología filosófica. Hacia un personalismo analógico-icónico. Salamanca, España: Editorial Fundación Emmanuel Mounier,.

Camus, A. (2002) La Peste. España: Edhasa

Greene, M. (2005) Liberar la imaginación. Ensayos sobre educación, arte y cambio social. España: Editorial GRAÓ, de IRIF, S.I.

Maturana, H. (2003) Emociones y lenguaje de la educación y la política. Brasil: Editorial UFMG

Saramago, J. (2004) Ensayo sobre la ceguera. Bogotá, Colombia: Editorial Nomos S.A.

Silva, L. (2011) Belleza y Revolución. Caracas, Venezuela: Fondo editorial Fundarte. 